Homology, Homotopy and Applications, vol.19(1), 2017, pp.371-389

\title{
BOUSFIELD LOCALIZATION OF GHOST MAPS
}

\author{
MARK HOVEY AND KEIR LOCKRIDGE
}

(communicated by J. Daniel Christensen)

\begin{abstract}
In homotopy theory, a ghost map is a map that induces the zero map on all stable homotopy groups. Bousfield localization is the homotopy-theoretic analogue of localization for rings and modules. In this paper, we consider the Bousfield localization of ghost maps. In particular, we pose the question: for which localization functors is it the case that the localization of a ghost is always a ghost? On the category of $p$-local spectra, we conjecture that the only localizations satisfying this property are the zero functor, the identity functor, and localization with respect to the rational Eilenberg-Mac Lane spectrum $H \mathbb{Q}$. We significantly narrow the field of possible counter-examples (one interesting outstanding possibility is the Brown-Comenetz dual of the sphere) and we consider a weaker version of the question at hand.
\end{abstract}

\section{Introduction}

In algebra, localization is a useful tool for studying rings and their categories of modules. Often a given property of a ring holds if and only if it holds for the ring's localization at every prime, or perhaps maximal, ideal. The localization $R_{\mathfrak{p}}$ of a commutative ring $R$ at a prime ideal $\mathfrak{p}$ is a local ring whose maximal ideal is the localization of $\mathfrak{p}$; this provides access to the extensive theory of commutative local rings. The functor $(-)_{\mathfrak{p}}$ from the category of $R$-modules to the category of $R_{\mathfrak{p}}$-modules enjoys many desirable properties. In particular,

$$
M_{\mathfrak{p}} \cong R_{\mathfrak{p}} \otimes_{R} M,
$$

and localization is exact since $R_{\mathfrak{p}}$ is flat over $R$.

In stable homotopy theory, a common - and extraordinarily useful - analogue of algebraic localization is Bousfield localization. For every spectrum $E$, there is an associated Bousfield localization functor $L=L_{E}$ that inverts $E$-homology equivalences and kills $E$-homology acyclics (see [Bou79] for details). For every spectrum $X$, there is a cofiber sequence

$$
C_{L} X \stackrel{j}{\longrightarrow} X \stackrel{i}{\longrightarrow} L X
$$

such that $i$ is an $E$-equivalence that is weakly initial among $E$-equivalences out of $X$.

Received February 26, 2016, revised July 7, 2016; published on May 17, 2017.

2010 Mathematics Subject Classification: 55P42, 55P60.

Key words and phrases: Bousfield localization, ghost map, ghost-preserving localization.

Article available at http://dx.doi.org/10.4310/HHA.2017.v19.n1.a18

Copyright (c) 2017, Mark Hovey and Keir Lockridge. Permission to copy for private use granted. 
The spectrum $C_{L} X$ is $E$-acyclic, meaning $E \wedge C_{L} X=0$, and $j$ is weakly final among maps from an $E$-acyclic spectrum into $X$. A spectrum is called $L$-local if it lies in the image of $L$.

In the ring $\pi_{*} S$, the stable homotopy groups of the sphere spectrum, the only non-trivial prime ideals are of the form $(p)$ for $p$ a prime integer. For each such ideal there is a Bousfield localization functor $L_{(p)}$ that gives rise to its algebraic cousin: one has

$$
\pi_{*} L_{(p)} X=\mathbb{Z}_{(p)} \otimes \pi_{*} X
$$

for all spectra $X$. For a general Bousfield localization functor $L$, the relationship between $\pi_{*} X$ and $\pi_{*} L X$ can be far more mysterious, and such behavior is extremely rare. It is therefore also difficult in general to determine the effect of localization on maps of spectra on the level of homotopy groups.

The concern of this paper is what Bousfield localizations do to ghost maps. A map $g$ is called a ghost if $\pi_{*} g=g_{*}=0$. An important open question in homotopy theory pertains to the existence of ghost maps: Freyd's generating hypothesis [Fre66] states that any ghost map between finite spectra is null homotopic. However, ghost maps involving infinite spectra abound. They play a central role in the homological algebra of modules over a ring spectrum, for example (see [HL09, HL11, HL13]). For a functor such as $L_{(p)}$, it is clear by (1) that the localization of a ghost must always be a ghost. We suspect that these are essentially the only such localizations, and this paper grew out of an attempt to prove it.

Fix a localization functor $L$. We say that a spectrum $X$ is $L$-ghost-preserving if the $L$-localization of every ghost with source $X$ is also a ghost. Refer to $L$ as ghost-preserving if all spectra are $L$-ghost-preserving, and call $L$ weakly ghostpreserving if all finite spectra are $L$-ghost-preserving. We begin by establishing some general facts about $L$-ghost-preserving spectra in $\S 2$. In that section, we work in the derived category of an $S$-algebra $R$. This is with a view toward the study of ghostpreserving localizations in other stable homotopy categories; however, the remainder of this paper concerns the case $R=S$. In $\S 3$, we observe that $p$-localization is ghostpreserving and $p$-completion is weakly ghost-preserving, but not ghost-preserving. In fact, localization with respect to any connective spectrum is weakly ghost-preserving. In $\S 4$, we relegate any non-obvious ghost-preserving localizations to the fringes of homotopy theory (Proposition 4.3). We eliminate all ring spectra other than $S$ and $H \mathbb{Q}$, but we do not know, for example, whether $L_{\mathbb{I}}$ is ghost-preserving, where $\mathbb{I}$ is the Brown-Comenetz dual of the sphere. Our arguments make use of nilpotence technology and the Bousfield lattice. In $\S 5$, we prove that $L_{n}$ is not weakly ghost-preserving for $n \geqslant 1$, and in $\S 6$, we prove that $L_{K(1)}$ is not weakly ghost-preserving. Though we suspect that $K(n)$-localization is not weakly ghost-preserving for $n>1$, we are not yet able to prove it. In $\S 7$, we briefly consider spectra $X$ such that the localization of any ghost into $X$ is a ghost. This is automatically a more difficult task, as it seems to require one to work with injective spectra, which are not well understood.

\section{Acknowledgments}

The authors would like to thank the referee for making many helpful suggestions and observations which improved the exposition in $\S 2$ and $\S 3$, streamlined the proof 
of the main theorem in $\S 4$, and simplified the proof of Proposition 5.2.

\section{Generalities}

Let $R$ be an $S$-algebra. In this section, we work in $\mathcal{D}(R)$, the derived category of left $R$-module spectra [EKMM97]. There are Bousfield localizations

$$
L=L_{E}: \mathcal{D}(R) \longrightarrow \mathcal{D}(R),
$$

for any right $R$-module spectrum $E$. The localization of an $S$-algebra is again an $S$-algebra, and the localization of a left $R$-module is a left $L R$-module. The category of $L$-local $R$-modules is equivalent to the full subcategory of $\mathcal{D}(L R)$ of objects that are $L$-local as $R$-modules. We will write $\mathcal{D}(R)(X, Y)$ for the set of $R$-module maps from $X$ to $Y$, and $\pi_{*}(-)=[S,-]_{*}=\mathcal{D}(R)(R,-)$. The observations and definitions made in $\S 1$ apply in this context. All $R$-modules are left modules unless otherwise indicated.

Every localization $L$ comes equipped with a natural transformation $i$ from the identity functor to $L$. For any $R$-module $X$, let $i$ also denote the localization map $i_{X}: X \longrightarrow L X$. This localization map induces an isomorphism

$$
i^{*}: \mathcal{D}(R)(L X, L Y) \longrightarrow \mathcal{D}(R)(X, L Y),
$$

for all modules $Y$. For any map $g: X \longrightarrow Y$, we have $i^{*} L g=(L g) i=i g$. Hence,

$$
i g \simeq 0 \text { if and only if } L g \simeq 0 .
$$

Certainly, $i^{*}$ preserves ghosts: if $h: L X \longrightarrow L Y$ is a ghost, then so is $i^{*} h=h i$. In particular, if $L g$ is a ghost, then $i g$ is a ghost. The converse is always true for ghostpreserving localizations (see Proposition 2.1 below).

An $R$-module $P$ is projective if and only if $P$ is a retract of a coproduct of suspensions of $R$, if and only if $P_{*}$ is a projective $R_{*}$-module. Further, every projective $R_{*}$-module is realizable as the homotopy of a projective module, and whenever $P$ is projective, $\mathcal{D}(R)(P, Y) \cong \operatorname{Hom}_{R_{*}}\left(P_{*}, Y_{*}\right)$ via the obvious natural map. See [HL13] for details. Now, let $\eta_{X}: P_{X} \longrightarrow X$ be a map out of a projective module such that $\pi_{*} \eta_{X}$ is surjective. Form the cofiber sequence

$$
P_{X} \stackrel{\eta_{X}}{\longrightarrow} X \stackrel{\Phi_{X}}{\longrightarrow} C_{X}
$$

To standardize this choice, one could take $\eta_{X}=\vee \eta_{\alpha}$, where $\left\{\eta_{\alpha}\right\}$ is the set of homogeneous elements in $X_{*}$. The map $\Phi_{X}$ is called a universal ghost out of $X$.

Proposition 2.1. Let $L$ be a localization functor, and fix an $R$-module spectrum $X$. The following statements are equivalent:

A. The module $X$ is L-ghost-preserving.

B. For all maps $g: X \longrightarrow Y$, the map ig is a ghost if and only if $L g$ is a ghost.

C. For all maps $g: X \longrightarrow Y$ with L-local target, the map $g$ is a ghost if and only if $L g$ is a ghost.

D. The map $L \Phi_{X}$ is a ghost. 
E. For all objects $Y$, the isomorphism $i^{*}$ from (2) induces an isomorphism

$$
i^{*}: \mathcal{D}(R)(L X, L Y)^{g h} \longrightarrow \mathcal{D}(R)(X, L Y)^{g h},
$$

where $\mathcal{D}(R)(A, B)^{\text {gh }}$ denotes the set of ghost maps from $A$ to $B$.

Consequently, $L$ is ghost-preserving if and only if the above statements are true for all $X$, and $L$ is weakly ghost-preserving if and only if the above statements are true for all finite $X$.

Proof. To see that $(\mathrm{A}) \Longrightarrow(\mathrm{B})$, observe that if $i g$ is a ghost, then it is a ghost out of $X$, so $L(i g)=L g$ is a ghost by $(\mathrm{A})$. The implication $(\mathrm{B}) \Longrightarrow(\mathrm{C})$ follows from the fact that localization is idempotent, and therefore $i g=g$ in $(\mathrm{C})$. For the implication $(\mathrm{C}) \Longrightarrow(\mathrm{D})$, note that $\left(L \Phi_{X}\right) i=i \Phi_{X}$ is a ghost to an $L$-local spectrum since $\Phi_{X}$ is a ghost, so $L\left(\left(L \Phi_{X}\right) i\right)=L \Phi_{X}$ is a ghost by (C).

Turning to the implication $(\mathrm{D}) \Longrightarrow(\mathrm{E})$, we have already observed that $i^{*}$ preserves ghosts. Consider $h: L X \longrightarrow L Y$ and suppose $i^{*} h=h i$ is a ghost. Then, $h i \eta_{X}=$ 0 , and there is a factorization $h i=k \Phi_{X}$, where $k: C_{X} \longrightarrow L Y$. Localizing, we obtain

$$
h=L h=L(h i)=L\left(k \Phi_{X}\right)=L k L \Phi_{X} .
$$

Consequently, if $L \Phi_{X}$ is a ghost, then $h$ is a ghost as well.

Finally, we prove that $(\mathrm{E}) \Longrightarrow(\mathrm{A})$. Assume $(\mathrm{E})$ holds and let $g: X \longrightarrow Y$ be a ghost map. Then, $i_{Y} g=(L g) i_{X}=i^{*} L g$ is also a ghost. By (E), $L g$ is a ghost as well. This completes the proof.

Remark 2.2. Since any ghost out of a projective module is trivial, it follows that the projective modules are $L$-ghost-preserving for any $L$. This means that $L$ is weakly ghost-preserving if and only if the class of finite $L$-ghost-preserving spectra is thick, and $L$ is ghost-preserving if and only if the class of $L$-ghost-preserving spectra is localizing.

Remark 2.3. It is true by definition that if $L$ is not weakly ghost-preserving, then it is not ghost-preserving. But it is perhaps worth pointing out that if there is a finite spectrum $X$ that is not $L$-ghost-preserving, then there is an infinite spectrum that is not $L$-ghost-preserving, namely an infinite coproduct of copies of $X$. This observation follows from the fact that the retract of an $L$-ghost-preserving spectrum is again $L$-ghost-preserving.

For any spectrum $X$, consider the following composite of evident $\pi_{*} L R$-module maps:

$$
\pi_{*} L R \otimes_{\pi_{*} R} \pi_{*} X \stackrel{\mu_{X}}{\longrightarrow} \pi_{*}\left(L R \wedge_{R} X\right) \stackrel{\nu_{X}}{\longrightarrow} \pi_{*} L X .
$$

The restriction of $\nu_{X} \mu_{X}$ to $\pi_{*} X=1 \otimes_{\pi_{*} R} \pi_{*} X$ is precisely $i_{*}$, so since $i \Phi_{X}=\left(L \Phi_{X}\right) i$ is a ghost, we have $\left(L \Phi_{X}\right)_{*} \nu_{X} \mu_{X}=0$. We say that $X$ is $L$-dense if $\nu_{X} \mu_{X}$ is surjective. If $X$ is $L$-dense, then $L \Phi_{X}$ is a ghost, so $X$ is $L$-ghost-preserving by Proposition 2.1. This establishes the following proposition.

Proposition 2.4. Let $L$ be a localization functor. Every $L$-dense $R$-module is $L$ ghost-preserving. In particular, if every $R$-module is $L$-dense, then $L$ is ghost-preserving, and if every finite $R$-module is $L$-dense, then $L$ is weakly ghost-preserving. 
Though not every $L$-ghost-preserving spectrum is $L$-dense in general (see Remark 2.10), it is useful to isolate situations where it suffices to find an object that is not $L$-dense in order to prove that $L$ is not ghost-preserving. The following proposition addresses objects that are as far away as possible from being $L$-dense.

Proposition 2.5. Let $L$ be a localization functor. If there exists a non-L-acyclic module $X$ such that $i: X \longrightarrow L X$ is a ghost, then $X$ is not L-ghost-preserving, so $L$ is not ghost-preserving. If $X$ is also finite, then $L$ is not weakly ghost-preserving.

Proof. The module $X$ in the statement of the proposition is not $L$-ghost-preserving because $L i=\mathbf{1}_{L X} \neq 0$ is not a ghost.

Example 2.6. The ghost-preserving localization of a ghost must also be a ghost, but this is not true in general. The localization map $i: H \mathbb{Z} / p^{\infty} \longrightarrow\left(H \mathbb{Z} / p^{\infty}\right)_{p}^{\wedge}$ is a ghost, yet $L i \simeq \mathbf{1}_{\left(H \mathbb{Z} / p^{\infty}\right)_{p}}$ is not a ghost since $\left(H \mathbb{Z} / p^{\infty}\right)_{p}^{\wedge}=\Sigma H \mathbb{Z}_{p}^{\wedge} \neq 0$ (see Proposition 3.1(B) for more details).

We also have the following two propositions concerning smashing localizations. A localization functor $L$ is smashing if the natural map

$$
L R \wedge_{R} X \longrightarrow L X
$$

is an equivalence for all $X$ (note that this map induces $\nu_{X}$ above). $L$ is smashing if and only if $L$ preserves coproducts (see [HPS97, 3.3.2]; the proof there is valid in this context), if and only if every object in $\mathcal{D}(L R)$ is $E$-local as an $R$-module [EKMM97, VIII.3]. Call $L$ weakly smashing if the map (4) is an isomorphism for all projective $R$-modules. If $L$ is weakly smashing, it follows that the map (4) is an isomorphism for all objects in the thick subcategory generated by the projective modules. This is the collection of all objects of finite projective dimension. If $X$ has finite projective dimension, then any sufficiently long sequence of ghosts out of $X$ will be null. In the stable category, there are spectra of infinite projective dimension (for example, there are arbitrarily long products of Steenrod operations that are non-trivial). If $R$ has finite global dimension, then weakly smashing localizations are also smashing.

Proposition 2.7. Let $L$ be a localization functor. The following statements are equivalent:

A. The functor $L$ is weakly smashing.

$B$. Every projective $R$-module is L-dense.

$C$. Every free $R$-module is $L$-dense.

$D$. The functor $L$ takes free $R$-module spectra to free $L R$-module spectra.

Proof. First, we prove that (A) and (B) are equivalent.

Let $P$ be a projective $R$-module and suppose $L$ is weakly smashing. The map $\mu_{P}$ is an isomorphism since $P$ is projective and the map $\nu_{P}$ is an isomorphism since $L$ is weakly smashing. This implies that $P$ is $L$-dense.

Conversely, suppose that every projective module is $L$-dense. To prove that $L$ is weakly smashing, it suffices to prove that (4) is an equivalence for coproducts of suspensions of $R$ (i.e., for free $R$-modules) since localization commutes with finite coproducts. Let $P=\bigvee_{\alpha} \Sigma^{d_{\alpha}} R$ be such a coproduct. The map $\mu_{P}$ is an isomorphism, 
and since $P$ is $L$-dense, $\nu_{P}$ is surjective. To complete the proof, we must show that $\nu_{P}$ is injective. In what follows, we will ignore the suspensions in $P$ to make the notation less complicated. Consider the diagram

$$
\bigvee_{\alpha} L R \stackrel{\kappa}{\longrightarrow} L\left(\bigvee_{\alpha} L R\right) \stackrel{j}{\longrightarrow} \prod_{\alpha} L R
$$

The localization map $\kappa$ is precisely the map (4), and $\kappa_{*}=\nu_{P}$. The map $j$ is determined by the natural map from the coproduct to the product which must factor through $\kappa$ since a product of $L$-local modules is $L$-local. The map $\kappa_{*}=\nu_{P}$ is injective since $j \kappa$ induces the natural injection

$$
\pi_{*} j \kappa: \bigoplus_{\alpha} \pi_{*} L R \longrightarrow \prod_{\alpha} \pi_{*} L R .
$$

Hence $\kappa$ is an equivalence, as desired.

That (B) implies (C) is obvious. To see that (C) implies (B), simply use the fact that the localization commutes with finite coproducts.

Statement (A) implies statement (D) by definition of weakly smashing, so it remains to show that (D) implies (A). Given a coproduct $\bigvee_{\alpha} R$ for $\alpha$ in an index set $A$, we need to prove that $\bigvee_{\alpha} L R$ is $L$-local. It suffices to do this for index sets $A$ of sufficiently large cardinality, so assume $|A|>\left|\pi_{*} L R\right|$. By (D), the localization of this spectrum is a free $L R$-module $\bigvee_{\beta} L R$ for $\beta$ in an index set $B$. Since the localization map induces an injection

$$
\pi_{*} \bigvee_{\alpha} L R \longrightarrow \pi_{*} \bigvee_{\beta} L R
$$

(this was observed above), the cardinality of the source may not exceed the cardinality of the target. This forces $|B| \geqslant|A|$. But then since the coproduct $\bigvee_{\beta} L R$ is $L$-local, any retract is also $L$-local, so $\bigvee_{\alpha} L R$ is $L$-local, as desired.

As an example, we next prove that neither $p$-completion nor $K(1)$-localization is weakly smashing.

Proposition 2.8. Neither p-completion nor K(1)-localization is weakly smashing.

Proof. In both cases, it suffices to find a coproduct of spheres that is not $L$-dense. Consider the countable coproduct of spheres $\underset{k}{\bigvee} S$, where no copy of $S$ is suspended. The $p$-completion map factors as

$$
\bigvee_{k} S \longrightarrow \bigvee_{k} S_{p}^{\wedge} \longrightarrow\left(\bigvee_{k} S_{p}^{\wedge}\right)_{p}^{\wedge} \cong\left(\bigvee_{k} S\right)_{p}^{\wedge}
$$

On $\pi_{0}$, this map induces

$$
\bigoplus_{k} \mathbb{Z} \longrightarrow \bigoplus_{k} \mathbb{Z}_{p}^{\wedge} \longrightarrow \operatorname{Ext}\left(\mathbb{Z} / p^{\infty}, \bigoplus_{k} \mathbb{Z}_{p}^{\wedge}\right) \cong\left(\bigoplus_{k} \mathbb{Z}_{p}^{\wedge}\right)_{p}^{\wedge}
$$

(Ext-p-completion and completion coincide for abelian groups whose $p$-torsion has 
bounded order; see [MP12]), and the image of the second map in the above composite does not generate the target as a $\mathbb{Z}_{p}^{\wedge}$-module. A similar proof works for $K(1)$ localization, since

$$
\pi_{0}\left(\bigvee_{k} L_{K(1)} S\right)_{p}^{\wedge} \cong\left(\bigoplus_{k} \mathbb{Z}_{p}^{\wedge}\right)_{p}^{\wedge} \oplus \operatorname{Hom}\left(\mathbb{Z} / p^{\infty}, \bigoplus_{k} \mathbb{Z}_{p}^{\wedge}\right) \cong\left(\bigoplus_{k} \mathbb{Z}_{p}^{\wedge}\right)_{p}^{\wedge}
$$

Remark 2.9. Any finite projective module is automatically $L$-dense for any $L$, because localization commutes with finite coproducts. This is little help in studying weakly ghost-preserving localizations when $R=S$, however, since there are no known examples of non-projective finite spectra $X$ such that $X_{*}$ is finitely generated, and if the generating hypothesis is true, then no such examples exist. Any of the statements in Proposition 2.7 imply that $L$ takes projective $R$-modules to projective $L R$-modules, but we do not know whether the converse is true. We also do not know whether there are weakly smashing localizations that are not smashing.

Remark 2.10. One consequence of Proposition 2.7 is that the converse of Proposition 2.4 is not true in general. For if every $L$-ghost-preserving spectrum were $L$-dense, then the projective spectra would always be $L$-dense, and every localization functor would be weakly smashing. This is not true, by Proposition 2.8.

Proposition 2.11. If $L$ is a weakly smashing localization, then a spectrum $X$ is $L$ ghost-preserving if and only if it is L-dense. In particular, if $L$ is weakly smashing, then

A. $L$ is ghost-preserving if and only if every spectrum is L-dense.

B. $L$ is weakly ghost-preserving if and only if every finite spectrum is L-dense.

Note that the previous two statements apply to smashing localizations.

Proof. In light of Proposition 2.4, it suffices to prove that every $L$-ghost-preserving spectrum is $L$-dense. Suppose $X$ is $L$-ghost-preserving. By localizing the cofiber sequence in (3), we obtain a cofiber sequence

$$
L P_{X} \stackrel{L \eta_{X}}{\longrightarrow} L X \stackrel{L \Phi_{X}}{\longrightarrow} L C_{X}
$$

where $L \Phi_{X}$ is a ghost and $L \eta_{X}$ is $\pi_{*}$-surjective. We now have the following commutative diagram:

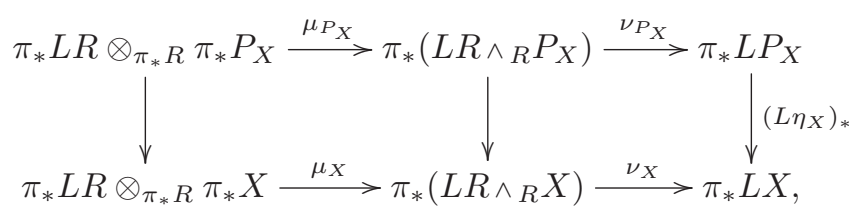

where $\left(L \eta_{X}\right)_{*}$ is surjective, $\mu_{P_{X}}$ is an isomorphism since $P_{X}$ is projective, and $\nu_{P_{X}}$ is an isomorphism since $P_{X}$ is $L$-dense (see Proposition 2.7). This implies that $\nu_{X} \mu_{X}$ is surjective, so $X$ is $L$-dense, as desired.

In the next few sections, we work entirely with the case $R=S$; i.e., we work in the usual stable homotopy category of spectra. 


\section{Moore spectra}

We have two results concerning Moore spectra and a corollary for connective spectra. Localization with respect to a Moore spectrum $M G$ is determined by the acyclicity type of $G$. Any abelian group $G$ has the acyclicity type of either $G=\mathbb{Z}_{(J)}$ or $G=\bigoplus_{p \in J} \mathbb{Z} /(p)$ for some set of primes $J$. Call localization with respect to the Moore spectrum $M \mathbb{Z}_{(J)} J$-localization, and let $X_{(J)}$ denote the $J$-localization of $X$. Call localization with respect to the Moore spectrum $M\left(\bigoplus_{p \in J} \mathbb{Z} /(p)\right) J$-completion, and let $X_{J}^{\wedge}$ denote the $J$-completion of $X$. Note that $J$-localization includes rationalization (localization with respect to $M \mathbb{Q}=H \mathbb{Q}$, where $H \mathbb{Q}$ is the rational EilenbergMac Lane spectrum).

Proposition 3.1. Let $J$ be a set of primes.

A. J-localization is ghost-preserving.

B. J-completion is weakly ghost-preserving, but not ghost-preserving.

$C$. If $E$ is a connective spectrum, then $L_{E}$ is weakly ghost-preserving.

Proof. First consider (A). By Proposition 2.4, it suffices to prove that every spectrum is $(-)_{J}$-dense. For any spectrum $X$,

$$
\pi_{*} X_{(J)} \cong \mathbb{Z}_{(J)} \otimes X_{*},
$$

where $\pi_{0} S_{(J)} \cong \mathbb{Z}_{(J)}$, so $\nu_{X} \mu_{X}$ is surjective and every spectrum is indeed $(-)_{J}$-dense.

For (B) it suffices to prove that every finite spectrum is $(-)_{p}^{\wedge}$-dense, again by Proposition 2.4. Any finite spectrum $X$ has finitely generated homotopy groups in each degree (though the converse is not true; consider $H \mathbb{Z}$, for example). Consequently,

$$
\pi_{*} X_{J}^{\wedge} \cong \pi_{*} \prod_{p \in J} X_{p}^{\wedge} \cong \prod_{p \in J} \pi_{*} X_{p}^{\wedge} \cong \prod_{p \in J}\left(\mathbb{Z}_{p}^{\wedge} \otimes X_{*}\right) \cong\left(\prod_{p \in J} \mathbb{Z}_{p}^{\wedge}\right) \otimes X_{*} .
$$

The first and third isomorphisms come from [Bou79]. The last isomorphism uses the fact that $\pi_{k} X$ is finitely presented for each integer $k$. Now, $\pi_{0} S_{J}^{\wedge} \cong \prod_{p \in J} \mathbb{Z}_{p}^{\wedge}$, and $\nu_{X} \mu_{X}$ is surjective, so every finite spectrum is indeed $(-)_{p}^{\wedge}$-dense.

To see that $J$-completion is not ghost-preserving, we first show that $p$-completion is not ghost-preserving. From [Bou79, 2.5], we have a split short exact sequence

$$
0 \longrightarrow \operatorname{Ext}\left(\mathbb{Z} / p^{\infty}, \pi_{k} X\right) \longrightarrow \pi_{k} X_{p}^{\wedge} \longrightarrow \operatorname{Hom}\left(\mathbb{Z} / p^{\infty}, \pi_{k-1} X\right) \longrightarrow 0 .
$$

Take $X=H \mathbb{Z} / p^{\infty}$, the Eilenberg-Mac Lane spectrum associated to $\mathbb{Z} / p^{\infty}$. Since

$$
\operatorname{Ext}\left(\mathbb{Z} / p^{\infty}, \mathbb{Z} / p^{\infty}\right)=0
$$

and

$$
\operatorname{Hom}\left(\mathbb{Z} / p^{\infty}, \mathbb{Z} / p^{\infty}\right) \cong \mathbb{Z}_{p}^{\wedge}
$$

we obtain that $\left(H \mathbb{Z} / p^{\infty}\right)_{p}^{\wedge} \cong \Sigma H \mathbb{Z}_{p}^{\wedge}$. Hence, the $p$-completion map

$$
H \mathbb{Z} / p^{\infty} \longrightarrow \Sigma H \mathbb{Z}_{p}^{\wedge}
$$

is a ghost, and $p$-completion is not ghost-preserving by Proposition 2.5. For any prime 
$q \neq p,\left(H \mathbb{Z} / p^{\infty}\right)_{q}^{\wedge}=0$, so $\left(H \mathbb{Z} / p^{\infty}\right)_{J}^{\wedge}=\left(H \mathbb{Z} / p^{\infty}\right)_{p}^{\wedge}$ for any $p \in J$, and the existence of the above ghost implies that $J$-completion is not ghost-preserving.

Finally, we turn our attention to (C). If $E$ is a connective spectrum, then for all connective spectra $X, L_{E} X \simeq L_{M G} X$, where $G$ has the acyclicity type of $E_{*}$ as an abelian group [Bou79, 3.1]. Since every finite spectrum is connective, every finite spectrum is $L_{E}$-dense if and only if every finite spectrum is $L_{M G}$-dense. Since every finite spectrum is $L_{M G}$-dense by the above proofs of (A) and (B), $L_{E}$ is weakly ghost-preserving by Proposition 2.4.

Remark 3.2. Another way to see that $J$-localization is ghost-preserving and $J$-completion is weakly ghost-preserving is to observe that, given the above calculations, in both cases $\pi_{*} L f$ has the form $\mathbf{1} \otimes f_{*}$, so if $f$ is a ghost then $L f$ is a ghost as well.

\section{Ghost-preserving localizations}

If $E$ is a spectrum, then

$$
L_{E_{(p)}}=L_{E} \circ(-)_{(p)}=(-)_{(p)} \circ L_{E} .
$$

A map $h$ is a ghost if and only if $h_{(p)}$ is a ghost for all primes $p$. Consequently, $L_{E}$ is ghost-preserving if and only if $L_{E_{(p)}}$ is ghost-preserving on the category of $p$-local spectra for all primes $p$. In this section, we assume all spectra are $p$-local. We have the following conjecture.

Conjecture 4.1. On the category of p-local spectra, the only ghost-preserving localizations are the zero functor, the identity functor, and $L_{H \mathbb{Q}}$.

We cannot prove this conjecture, but we are able to eliminate practically every other interesting possibility (see Proposition 4.3). (A notable exception is $L_{\mathbb{I}}$, localization with respect to the Brown-Comenetz dual of the $p$-local sphere.) This conjecture might be interpreted to say that the ghost preserving localizations coincide with the localizations which are 'purely algebraic,' giving rise to localization at a prime ideal of $\pi_{*} S$.

We will need to make use of the technology surrounding the Nilpotence Theorem; see [HS98, Hov95] for more details. Let $K(n)$ denote the $n$th Morava $K$-theory with $K(n)_{*}=\mathbb{F}_{p}\left[v_{n}, v_{n}^{-1}\right]$. These spectra are algebraic localizations of certain modules over the Brown-Petersen spectrum $B P$ with $B P_{*}=\mathbb{Z}_{(p)}\left[v_{1}, v_{2}, \ldots\right]$ and $\left|v_{n}\right|=2\left(p^{n}-1\right)$. If a $p$-local finite spectrum is $K(n)$-acyclic for some $n$, then it is $K(i)$-acyclic for all $i<n$. A finite spectrum has type $n$ if $n$ is the smallest non-negative integer such that $K(n)_{*} X \neq 0$, and every spectrum is type $n$ for some $n$. Let $\mathcal{C}_{n}$ denote the spectra of type at least $n$. There is a sequence of proper inclusions

$$
\cdots \subseteq \mathcal{C}_{n+1} \subseteq \mathcal{C}_{n} \subseteq C_{n-1} \subseteq \cdots \subseteq \mathcal{C}_{0},
$$

and the Nilpotence Theorem [HS98, Theorem 7] implies that every non-trivial thick subcategory of $\mathcal{C}_{0}$ is equal to $\mathcal{C}_{n}$ for some $n$. Every type $n$ spectrum determines the same Bousfield class $\langle F(n)\rangle$. The type $n$ spectrum $F(n)$ admits a $v_{n}$ self-map inducing an isomorphism on $K(m)_{*} F(n)$ if $m=n$ and zero otherwise. The Bousfield class $\langle\operatorname{Tel}(n)\rangle$ of the telescope of this map is independent of both the choice of $F(n)$ and the choice of $v_{n}$ self-map. Further, $K(m) \wedge \operatorname{Tel}(n)=0$ if $m \neq n$. 
We will also make use of the mod $(p)$ Eilenberg-Mac Lane spectrum $K(\infty)=H \mathbb{F}_{p}$ and $\mathbb{I}$, the Brown-Comenetz dual of the ( $p$-local) sphere. The Brown-Comenetz dual $\mathbb{I} X$ of a spectrum $X$ is the representing spectrum for the cohomology theory

$$
\operatorname{Hom}\left(\pi_{0}(X \wedge(-)), \mathbb{Q} / \mathbb{Z}_{(p)}\right) .
$$

Some relevant properties of $\mathbb{I}$ are summarized in $[\mathrm{HP99}, 7.1]$. In particular, $\left\langle H \mathbb{F}_{p}\right\rangle \geqslant$ $\langle I\rangle$. Our next goal is to prove Proposition 4.3, which narrows the class of possible ghost-preserving localizations. First, we have the following useful observation.

Proposition 4.2. Let $L_{E}$ be a ghost-preserving localization. If there exists a $\pi_{*}$ injective map $X \longrightarrow Y$ where $Y$ is E-acyclic, then $X$ is E-acyclic.

Proof. Let $L=L_{E}$ be a ghost-preserving localization and let $f: X \longrightarrow Y$ be $\pi_{*}$ injective. Since the fiber of $f$ is a ghost, and $L$ is ghost-preserving, the map $L f$ is $\pi_{*}$-injective. Hence, if $L Y=0$, then $L X=0$.

A spectrum $X$ is dissonant if it is acyclic with respect to the wedge of Morava $K$-theories

$$
\bigvee_{0 \leqslant n<\infty} K(n)
$$

Spectra that are local with respect to this wedge are called harmonic. See [Rav84] for a discussion of harmonic and dissonant spectra.

Proposition 4.3. Let $E$ be a spectrum and let $D=E \wedge M(p)$. If $L_{E} \neq L_{S}$ is ghostpreserving, then $D$ is a BP-acyclic dissonant spectrum and $\langle E\rangle=\langle D\rangle$ or $\langle E\rangle=$ $\langle D\rangle \vee\langle H \mathbb{Q}\rangle$.

Proof. In general,

$$
\langle E\rangle=\langle E \wedge H \mathbb{Q}\rangle \vee\langle E \wedge M(p)\rangle .
$$

So $\langle E\rangle=\langle D\rangle$ if $E \wedge H \mathbb{Q}=0$ and $\langle E\rangle=\langle H \mathbb{Q}\rangle \vee\langle D\rangle$ otherwise.

First, suppose $E \wedge H \mathbb{Q}=0$. The rationalization map $B P \longrightarrow B P \wedge H \mathbb{Q}$ is $\pi_{*^{-}}$ injective and $E \wedge B P \wedge H \mathbb{Q}=0$, so $E \wedge B P=0$ by Proposition 4.2. This implies that $E \wedge A=0$ for the $B P$-module spectra $A=K(n)$ and $A=H \mathbb{F}_{p}$. In particular, $E$ and $E \wedge M(p)$ are BP-acyclic dissonant spectra, $\langle E\rangle=\langle D\rangle$, and $E \wedge H \mathbb{F}_{p}=0$.

Next, suppose $E \wedge H \mathbb{F}_{p} \neq 0$. By the previous paragraph, $E \wedge H \mathbb{Q} \neq 0$. Since $H \mathbb{F}_{p}$ and $H \mathbb{Q}$ are both field spectra, we then have $\langle E\rangle \geqslant\langle H \mathbb{Q}\rangle \vee\left\langle H \mathbb{F}_{p}\right\rangle=\left\langle H \mathbb{Z}_{(p)}\right\rangle$. For any spectrum $X$, the Postnikov cover $X[d, \infty) \longrightarrow X$ is $\pi_{*}$-injective. If $E \wedge X=0$, then $E \wedge X[d, \infty)=0$ by Proposition 4.2. This forces $H \mathbb{Z}_{(p)} \wedge X[d, \infty)=0$, so $\pi_{d} X=0$. Since $d$ was arbitrary, this forces $\pi_{*} X=0$ and hence $X$ is trivial. Since the trivial spectrum is the only $E$-acyclic spectrum, $L_{E}$ is the identity functor.

Finally, suppose $E \wedge H \mathbb{F}_{p}=0$ and $E \wedge H \mathbb{Q} \neq 0$. We will now make use of the $B P$-module (and ring spectrum) $B P\langle 1\rangle$ with coefficient ring $B P\langle 1\rangle_{*}=\mathbb{Z}_{(p)}\left[v_{1}\right]$. By [Rav84, 2.1(f)],

$$
\langle B P\langle 1\rangle\rangle=\langle H \mathbb{Q}\rangle \vee\langle K(1)\rangle \vee\left\langle H \mathbb{F}_{p}\right\rangle .
$$

Smashing with $E$, we obtain that $\langle E \wedge B P\langle 1\rangle\rangle=\langle H \mathbb{Q}\rangle$ or

$$
\langle E \wedge B P\langle 1\rangle\rangle=\langle H \mathbb{Q}\rangle \vee\langle K(1)\rangle=\langle E(1)\rangle .
$$

We claim that $\langle E \wedge B P\langle 1\rangle\rangle \neq\langle E(1)\rangle$; to prove this, we use the following lemma. 
Lemma. If $T$ is a ring spectrum, then $L_{E} M=L_{E \wedge T} M$ for all left $T$-module spectra $M$.

Proof. Certainly, $\langle E\rangle \geqslant\langle E \wedge T\rangle$, so $L_{E \wedge T} M$ is $E$-local for any $M$. If $M$ is a left $T$-module, then the map

$$
E \wedge M \stackrel{E \wedge i}{\longrightarrow} E \wedge L_{E \wedge T} M
$$

is a retract of the equivalence

$$
E \wedge T \wedge M \frac{E \wedge T \wedge i}{\approx} E \wedge T \wedge L_{E \wedge T} M
$$

via the module structure maps (both $M$ and $L_{E \wedge T} M$ are left $T$-module spectra). The map $E \wedge i$ is therefore an equivalence, making $i$ and $E$-equivalence, so $L_{E \wedge T} M=$ $L_{E} M$ as desired.

The rationalization map $f: B P\langle 1\rangle \longrightarrow B P\langle 1\rangle \wedge H \mathbb{Q}$ is a $\pi_{*}$-injective map of left $B P\langle 1\rangle$-modules, so by the lemma $L_{E} f=L_{E \wedge B P\langle 1\rangle} f$ is $\pi_{*}$-injective since $L_{E}$ is ghostpreserving. However, $L_{E(1)} f$ is not $\pi_{*}$-injective since

$$
L_{E(1)} B P\langle 1\rangle \wedge H \mathbb{Q}=B P\langle 1\rangle \wedge H \mathbb{Q}
$$

is connective and $L_{E(1)} B P\langle 1\rangle$ is not connective. Therefore $\langle E \wedge B P\langle 1\rangle\rangle=\langle H \mathbb{Q}\rangle$ and $E \wedge K(1)=0$. Now, since $\left\langle v_{1}^{-1} B P\right\rangle=\langle H \mathbb{Q}\rangle \vee\langle K(1)\rangle$, we have $\left\langle E \wedge v_{1}^{-1} B P\right\rangle=\langle H \mathbb{Q}\rangle$ and thus

$$
E \wedge v_{1}^{-1} B P \wedge M(p)=0
$$

Since the map $B P \longrightarrow v_{1}^{-1} B P$ is $\pi_{*}$-injective, Proposition 4.2 implies that

$$
E \wedge M(p) \wedge B P=0 .
$$

This means $E \wedge M(p)$ is a $B P$-acyclic dissonant spectrum, and the proof of the proposition is complete.

Remark 4.4. Note that $E \neq S$ in the above proposition cannot be a ring spectrum, for if $E$ were a ring spectrum then $E \wedge M(p)$ would be a dissonant, $H \mathbb{F}_{p}$-acyclic ring spectrum, but every ring spectrum is detected by some Morava $K$-theory.

Given a $p$-local spectrum $E$, let

$$
\mathrm{FA}(E)=\{X \mid X \text { is finite and } E \wedge X=0\}
$$

denote the finite acyclics of $E$. One of the equivalent forms of the Telescope Conjecture (believed to be false for $n \geqslant 2$, but true for $n=0,1)$ is that $\langle\operatorname{Tel}(n)\rangle=\langle K(n)\rangle$.

Proposition 4.5. Assume the Telescope Conjecture is true for all n. Let $E$ be a nontrivial p-local spectrum with $\mathrm{FA}(E) \neq 0$. If $L_{E}$ is ghost-preserving, then $\langle E\rangle=\langle H \mathbb{Q}\rangle$. 
Proof. By assumption, $\mathrm{FA}(E)=\mathcal{C}_{n+1}$ for some $n \geqslant 0$. As observed in [Hov95], repeated application of $[\operatorname{Rav} 84,1.34]$ yields

$$
\langle S\rangle=\langle\operatorname{Tel}(0)\rangle \vee \cdots \vee\langle\operatorname{Tel}(n)\rangle \vee\langle F(n+1)\rangle .
$$

Consequently,

$$
\langle E\rangle=\bigvee_{i=0}^{n}\langle E \wedge \operatorname{Tel}(i)\rangle
$$

because $F(n+1)$ is $E$-acyclic. If the Telescope Conjecture is true, then $\langle\operatorname{Tel}(n)\rangle=\langle K(n)\rangle$. By Proposition 4.3, we obtain $\langle E\rangle=\langle E \wedge H \mathbb{Q}\rangle=\langle H \mathbb{Q}\rangle$ since $E$ is non-trivial.

Since the telescope conjecture is true for $n=1$, we may conclude that for $E$ in the proposition, if $\langle E\rangle \neq\langle H \mathbb{Q}\rangle$, then $\mathrm{FA}(E) \subseteq \mathcal{C}_{3}$. In sum, it seems that the only ( $p$-local) examples of ghost-preserving localizations are $L=0, L_{S}$ and $L_{H \mathbb{Q}}$, though we have not proved this. Noteworthy among the outstanding possibilities is localization with respect to $\mathbb{I}$. We conclude this section with a general statement about weakly ghost-preserving localizations, which are far more common (recall that localization with respect to a connective spectrum is always weakly ghost-preserving by Proposition $3.1(\mathrm{C})$ ).

Proposition 4.6. If $E$ is a p-local spectrum with a non-trivial, finite, L-local object (e.g., a ring spectrum with no finite acyclics), then $E$ is weakly ghost-preserving.

Proof. By [Hov95, 3.7], $L_{E}$ must be the identity on finite spectra if $E \wedge H \mathbb{Q} \neq 0$, and $p$-completion on finite spectra otherwise. In the proof of [Hov95, 3.6] it is shown that a ring spectrum without finite acyclics has a finite local object.

In particular, $L_{\mathbb{I}}$ is weakly ghost-preserving.

\section{5. $E(n)$-localization}

Proposition 4.3 implies that the functor $L_{n}$ (localization with respect to $K(0) \vee \cdots$ $\vee K(n))$ is not ghost-preserving for $n \geqslant 1$. This is the same as $E(n)$-localization, where $E(n)_{*}=\mathbb{Z}_{(p)}\left[v_{1}, \cdots, v_{n}, v_{n}^{-1}\right]$. (The spectrum $E(1)$ is a summand of (non-connective) $p$-local complex $K$-theory.) We will now prove that $L_{n}$ is not weakly ghost-preserving for $n \geqslant 1$. We will make use of the following analogue of Nishida's Theorem.

Proposition 5.1. In the rings $\pi_{*} L_{n} S$ and $\pi_{*} L_{K(n)} S$, every element of non-zero degree is nilpotent.

Proof. First, we consider $\pi_{*} L_{n} S$. Since $L_{n} S$ is a ring spectrum, it suffices to prove that every self-map $f: L_{n} S \longrightarrow L_{n} S$ of non-zero degree is nilpotent. To do so, we use [HS99, 6.6], which says that $f$ is nilpotent if and only if $K(i)_{*} f$ is nilpotent for all $i \leqslant n$. Since $K(i)_{*} L_{n} S=K(i)_{*}$ for $i \leqslant n, f$ is nilpotent if and only if $K(i)_{*} f=0$ for all $i \leqslant n$, if and only if the cofiber $X$ of $f$ is not $K(i)$-acyclic for all $i \leqslant n$. Since $f$ has positive degree, $K(0)_{*} f=0$, so $X$ is not $K(0)$-acyclic and hence not $K(i)$-acyclic for any $i \leqslant n$ by [HS99, 6.8]. Thus $f$ is nilpotent.

For $\pi_{*} L_{K(n)} S$, we take a different approach that makes slight use of a spectral sequence converging to it; see [DH04, DH95] for more details. Let $E_{n}$ denote Morava 
$E$-theory, with $E_{n *}=W \mathbb{F}_{p^{n}}\left[\left[u_{1}, \ldots, u_{n-1}\right]\right]\left[u, u^{-1}\right]$. The ring $W \mathbb{F}_{p^{n}}$ is the ring of Witt vectors with coefficients in $\mathbb{F}_{p^{n}}$, and the degrees of the coordinates are $\left|u_{i}\right|=0$ and $|u|=-2$. There is an action of the Morava stabilizer group $S_{n}$ on $E_{n *}$ and a spectral sequence

$$
H_{c}^{*}\left(S_{n}, E_{n *}\right)^{\mathrm{Gal}} \Longrightarrow \pi_{*} L_{K(n)} S .
$$

Here, the Galois group Gal $=\operatorname{Gal}\left(\mathbb{F}_{p^{n}} / \mathbb{F}_{p}\right)$ of automorphisms of $\mathbb{F}_{p^{n}}$ acts trivially on the $u_{i}$ and $u$. This spectral sequence is isomorphic to the $K(n)$-local $E_{n}$-Adams spectral sequence from the $E_{2}$ page onward.

Any element $f \in \pi_{*} L_{K(n)} S$ of positive filtration induces the zero map in $E_{n^{-}}$ homology, hence its telescope is $E_{n}$-acyclic. Since $\left\langle E_{n}\right\rangle=\langle E(n)\rangle$, this telescope is a ring spectrum that is $K(i)$-acyclic for all $i$, hence trivial. Thus any element with positive filtration is nilpotent. It remains to consider maps with filtration zero. Such maps are detected by elements in $H_{c}^{0}\left(G_{n}, E_{n *}\right)$, the $G_{n}$-invariants of $E_{n *}$. To complete the proof, we need only prove that every such invariant has degree zero.

To do so, we will make slight use of [DH95, 3.3], where Devinatz and Hopkins describe an aspect of the action of $S_{n}$ on $E_{n *}$. The action there is given in terms of a different set of coordinates for $E_{n *}$, but if $t \in\left(\mathbb{Z}_{p}^{\wedge}\right)^{\times} \subseteq S_{n}$ is a $p$-adic unit (in the context of the proposition, $a_{0}=t$ and $a_{i}=0$ for $i=1, \ldots, n-1$ ), then $t$ acts as multiplication by $t^{k}$ in degree $-2 k$. This is enough to show that $E_{n *}$ has no invariants of non-zero degree, and the proof is complete.

Proposition 5.2. For $n \geqslant 1$, there exists a finite spectrum that is neither $L_{n}$-dense nor $L_{K(n)}$-dense.

Proof. Let $L=L_{n}$ or $L=L_{K(n)}$. For any $n \geqslant 1$, there is a type $n$ ring spectrum $V$ with $\pi_{k} V=0$ for $k<0$ [Dev92]. The ring spectrum $V$ admits a $v_{n}$-self map $\rho$ such that $K(n)_{*} \rho$ is an isomorphism and $K(i)_{*} \rho=0$ for $i<n$. Hence $L \rho$ is an invertible element of $\pi_{*} L V$ of non-zero degree. Let $J$ be the ideal of elements of $\pi_{*} L S$ of nonzero degree; by Proposition 5.1, $J$ is nilpotent. Consequently, $J \otimes_{\pi_{*} S} \pi_{*} V$ maps to zero via the composite

$$
\pi_{*} L S \otimes_{\pi_{*} S} \pi_{*} V \stackrel{\nu_{V} \mu_{V}}{\longrightarrow} \pi_{*} L V \longrightarrow K(n)_{*} L V .
$$

This composite now factors through the connective ring $\left(\pi_{*} L S\right) / J \otimes_{\pi_{*} S} \pi_{*} V$, so its image is connective. Thus, for $k$ sufficiently large, $(L \rho)^{-k}$ cannot be in the image of $\nu_{V} \mu_{V}$. This proves that the finite spectrum $V$ is not $L$-dense.

Corollary 5.3. The E(n)-localization functor $L_{n}$ is not weakly ghost-preserving for $n \geqslant 1$.

Proof. Since $L_{n}$ is smashing, it suffices to exhibit a finite spectrum that is not $L_{n^{-}}$ dense by Proposition 2.11. This was done above.

Since $L_{K(n)}$ is not smashing for $n \geqslant 1$, we cannot use the proposition to immediately conclude that $L_{K(n)}$ is not weakly ghost-preserving. However, we are able to prove this for $n=1$; see $\S 6$. 
Remark 5.4. Since the $n=1$ case is computationally accessible, we present it here. For $p$ an odd prime, let $M$ denote the $\bmod (p)$ Moore spectrum in the cofiber sequence

$$
S \stackrel{p}{\longrightarrow} S \stackrel{\iota}{\longrightarrow} M \stackrel{\partial}{\longrightarrow} \Sigma S .
$$

(For $p=2$, one would need to work with, say, the $\bmod (4)$ Moore spectrum.) We need:

Proposition 5.5 ([Mil81]). Let $\sigma=L_{1} \nu_{1} \iota$ and let $\rho=L_{1} \iota \alpha_{1}$. Note that $|\sigma|=q=$ $2(p-1)$ and $|\rho|=q-1$.

A. $\pi_{*} L_{1} M$ is ring isomorphic to $\mathbb{F}_{p}\left[\sigma, \sigma^{-1}\right] \otimes E(\rho)$.

$B$. The $\pi_{*} L_{1} S$-module structure is determined by the fact that, for $p \nmid s$, multiplication by $\alpha_{s p^{j} / j+1}$ acts as multiplication by $\sigma^{s p^{j}-1} \rho$ and $\pi_{-2} L_{1} S \cdot \pi_{*} L_{1} M=0$.

The map $\nu_{1}$ induces multiplication by $v_{1}$ in $B P$-homology, and $\alpha_{1}$ is the composite

$$
\Sigma^{2(p-1)} S \stackrel{\iota}{\longrightarrow} \Sigma^{2(p-1)} M \stackrel{\nu_{1}}{\longrightarrow} M \stackrel{\partial}{\longrightarrow} \Sigma S .
$$

See [Dev90, 1.1] (a reformulation of [Rav84, 8.10]) for a description of $\pi_{*} L_{1} S$ and the elements $\alpha_{s p^{j} / j+1}$, which generate $\pi_{s p^{j} q-1} L_{1} S \cong \mathbb{Z} /\left(p^{j+1}\right)$.

Now, $i_{*} M_{*}=\mathbb{F}_{p}[\sigma] \otimes E(\rho) \subseteq \pi_{*} L_{1} M$, and by Proposition 5.5, the $\pi_{*} L_{1} S$-submodule generated by $i_{*} M_{*}$ is $\mathbb{F}_{p}[\sigma] \otimes E(\rho) \cup \mathbb{F}_{p}\left[\sigma, \sigma^{-1}\right] \otimes \rho$. In particular, the submodule is proper and does not contain any elements of the form $\sigma^{k}$ for $k<0$. So $M$ is not $L_{1}$-dense, and $L_{1}$ is not weakly ghost-preserving.

We also give an explicit map $g$ such that $i g$ is a ghost, yet neither $g$ nor $L_{1} g=$ $L_{1}(i g)$ is a ghost. We prove that $L_{1} g$ is not a ghost here; it is proved that $g$ is not a ghost in [Dev90, 1.7], though our $g$ does not include the last map in the composite defined there. Note the use of $\sigma^{-1}$ below. Let $g=\alpha_{1} \partial$. Now, $\pi_{*} L_{1} g \neq 0$ (ignoring suspensions):

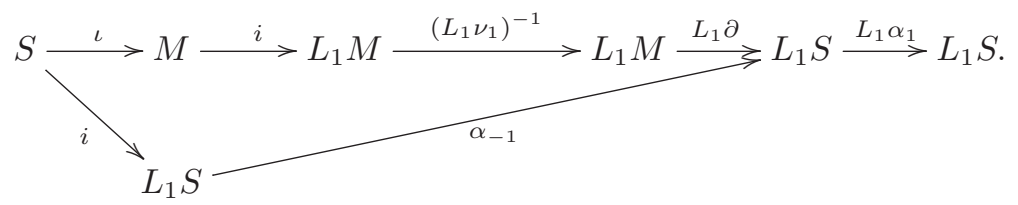

The bottom composition is non-zero by [Dev90, 1.1]; in $\pi_{*} L_{1} S$, the product of $\alpha_{1}$ and $\alpha_{-1}$ is an element of order $p$ in $\pi_{-2} L_{1} S \cong \mathbb{Q} / \mathbb{Z}_{(p)}$. Yet, $\pi_{*} i g=0$ (ignoring suspensions):

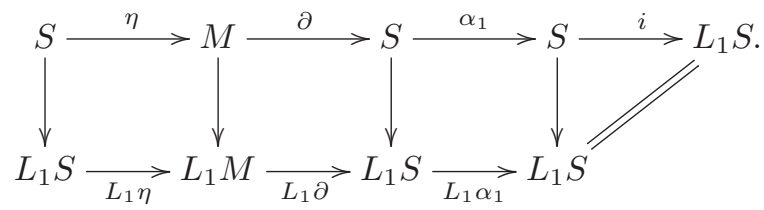

Without loss of generality, we may assume $|\eta| \geqslant 0$ since $\pi_{k} M=0$ for $k<0$. Now, $i \alpha_{1} \partial \eta=0$ if and only if $L_{1} \alpha_{1} \cdot L_{1}(\partial \eta)=0$. We have $\left|L_{1}(\partial \eta)\right| \geqslant-1$, but $\pi_{-1} L_{1} S=$ 0 , so, in fact, $\left|L_{1}(\partial \eta)\right| \geqslant 0$. But note that $L_{1}(\partial \eta)$ is $p$-torsion because $p \partial=0$, yet $\pi_{0} L_{1} S=\mathbb{Z}_{(p)}$ is torsion free. Consequently, $L_{1}(\partial \eta)$ has positive degree. The product of any such element with $L_{1} \alpha_{1}$ is zero. 


\section{Morava $K$-theories}

Next, we consider the $p$-completion of $L_{1}$, which is the same as $K(1)$-localization. In general, $\left(L_{n}(-)\right)_{p}^{\wedge}$ is not the same as $L_{K(n)}$. It is localization with respect to $K(1) \vee \cdots \vee K(n)$ : since $(-)_{p}^{\wedge}$ is localization with respect to the Moore spectrum of a torsion group, by [Bou79, 2.11] $\left(L_{n}(-)\right)_{p}^{\wedge}$ is the same as $E(n) \wedge M$-localization. Since $\langle E(n)\rangle=\langle K(0) \vee \cdots \vee K(n)\rangle$, this is further the same as localization with respect to

$$
(K(0) \wedge M) \vee \cdots \vee(K(n) \wedge M) .
$$

Now, $K(0) \wedge M=0$, and $K(n) \wedge M$ is a free $K(n)$-module spectrum, so $\left(L_{n}(-)\right)_{p^{-}}$ localization is the same as localization with respect to $K(1) \vee \cdots \vee K(n)$. We already know that neither $\left(L_{n}\right)_{p}^{\wedge}$ nor $L_{K(n)}$ is ghost-preserving by Proposition 4.3.

Our next goal is to prove that $K(1)$-localization is not weakly ghost-preserving. We will use the following description of $\pi_{*} L_{K(1)} S$, which follows from Ravenel's computation of $\pi_{*} L_{1} S$ in [Rav84, 8.10].

Proposition 6.1. Let $p$ be an odd prime. Then,

$$
\pi_{k}\left(L_{1} S\right)_{p}^{\wedge}=\pi_{k} L_{K(1)} S= \begin{cases}\mathbb{Z}_{p}^{\wedge} & \text { if } k=0,-1, \\ \mathbb{Z} / p^{i+1} \mathbb{Z} & \text { if } k=s p^{i} q-1, p \nmid s, \\ 0 & \text { otherwise. }\end{cases}
$$

The groups $\mathbb{Z} / p^{i+1} \mathbb{Z}$ are generated by the $p$-completions of the maps detected by the elements $\alpha_{s p^{i} / i+1}$ described in [Dev90, 1.1]. The group $\pi_{0} L_{1} S_{p}^{\wedge}$ is generated by the unit map. A non-zero element $h \in \pi_{-1}\left(L_{1} S\right)_{p}^{\wedge}$ is not in the image of the p-completion map; it corresponds to a map $h: \Sigma^{-1} M \mathbb{Z} / p^{\infty} \longrightarrow \Sigma^{1} L_{1} S$ such that $\pi_{-1} h \in \operatorname{Hom}\left(\mathbb{Z} / p^{\infty}, \mathbb{Z} / p^{\infty}\right) \cong \mathbb{Z}_{p}^{\wedge}$. The product of any two elements of non-zero degree is trivial, for degree reasons.

Proposition 6.2. At odd primes, the $K(1)$-localization functor $L_{K(1)}$ is not weakly ghost-preserving.

Proof. It is not necessary to work with the cofiber sequence containing a universal ghost out of $X$ in $\S 2$. One may instead work with a similar sort of map out of $L X$ (which will not be a universal ghost in general). Let $L=L_{K(1)}$. Ignoring suspensions, let $h$ be a surjection from a (necessarily infinite) coproduct $\bigvee S_{*}$ onto $i_{*} M_{*}$. The map $h$ lifts over the inclusion of $i_{*} M_{*}$ into $\pi_{*} L M$, and this lift is realizable as a map $\eta: \bigvee S \longrightarrow L M$. By construction, im $\eta_{*}=i_{*} M_{*}$. Now consider the cofiber sequence

$$
\bigvee S \stackrel{\eta}{\longrightarrow} L M \stackrel{\Phi_{M}^{\prime}}{\longrightarrow} C_{M}^{\prime}
$$

The map $\Phi_{M}^{\prime} i$ is a ghost, so it factors through $\Phi_{M}$, and if $L \Phi_{M}^{\prime}$ is not a ghost, then neither is $L \Phi_{M}$.

To prove that $L \Phi_{M}^{\prime}$ is not a ghost, we will prove that $\operatorname{im} \pi_{*} L \eta=\operatorname{im} \nu_{M} \mu_{M}$, which implies $\pi_{*} L \eta$ is not surjective by Proposition 5.2 (the proof shows that $M$ is not $K(1)$-dense since it is a type 1 ring spectrum). The infinite wedge $\bigvee S$ will not be $L$-dense (see the proof of Proposition 2.8), but since $p$ annihilates $\pi_{*} L M$ it suffices 
to prove that

$$
i_{*}: \pi_{*} \bigvee S \longrightarrow \pi_{*} L(\bigvee S)
$$

is surjective $\bmod (p)$. Now, $L(\bigvee S)=L(\bigvee L S)=(\bigvee L S)_{p}^{\wedge}$ since $\bigvee L S$ is already $E(1)$ local. The group $\pi_{*}(\bigvee L S)_{p}^{\wedge}$ has two summands:

$$
\operatorname{Ext}\left(\mathbb{Z} / p^{\infty}, \pi_{*} \bigvee L S\right)
$$

and

$$
\operatorname{Hom}\left(\mathbb{Z} / p^{\infty}, \pi_{*-1} \bigvee L S\right)=\operatorname{Hom}\left(\mathbb{Z} / p^{\infty}, \bigoplus \pi_{*-1} L S\right)
$$

The Hom group is zero: every summand of $\oplus \pi_{*-1} L S$ is a copy of either $\mathbb{Z}_{p}^{\wedge}$ or $\mathbb{Z} / p^{r}$ for some $r$, so it is the direct sum of a free abelian group and a $p$-torsion group that has no infinitely $p$-divisible elements. Consequently, the localization map induces Ext- $p$ completion

$$
\pi_{*} \bigvee L S \longrightarrow \pi_{*}(\bigvee L S)_{p}^{\wedge}=\operatorname{Ext}\left(\mathbb{Z} / p^{\infty}, \pi_{*} \bigvee L S\right)
$$

Though the image of this map will not generate the target as a $\pi_{*} L S$-module, this map is surjective $\bmod (p)$ : for any abelian group $A$, there is a surjection $\operatorname{Ext}\left(\mathbb{Z} / p^{\infty}, A\right) \longrightarrow$ $A_{p}^{\wedge}$ whose kernel consists of infinitely $p$-divisible elements, and so $\bmod (p)$ we have an isomorphism

$$
\operatorname{Ext}\left(\mathbb{Z} / p^{\infty}, A\right) /\left(p \operatorname{Ext}\left(\mathbb{Z} / p^{\infty}, A\right)\right) \cong\left(A_{p}^{\wedge}\right) / p\left(A_{p}^{\wedge}\right) \cong A / p A .
$$

This completes the proof.

Remark 6.3. The reader may wonder whether the map $f$ in Remark 5.4 may be used to show that $M$ is not $L_{K(1)}$-ghost-preserving. That map satisfies $\pi_{*}$ if $=0$, where $i$ is given by the localization $L_{K(1)}$, but unfortunately $\pi_{*} L_{K(1)} f=0$ as well: in order to have $\pi_{*} L_{K(1)} f \neq 0$, one would need an element of $\pi_{0} L_{1} S_{p}^{\wedge}$ (any element of non-zero degree would have a trivial product with $\left.\left(L_{1} \alpha_{1}\right)_{p}^{\wedge}\right)$ that factors through $\left(L_{1} \partial\right)_{p}^{\wedge}$; this would require a non-trivial element of $\pi_{1} L_{1} M_{p}^{\wedge}$, but this group is zero.

Remark 6.4. Here is an explicit computation that shows $M$ is not $L_{K(1)}$-dense. The spectrum $L_{1} M$ is already $p$-complete, so Proposition 5.5 gives the ring structure of $\pi_{*} L_{K(1)} M$. This proposition also gives the $\pi_{*} L_{K(1)} S$-module structure, with the exception of the action of $\pi_{-1} L_{K(1)} S$. The generator of this group acts as $\sigma^{-1} \rho$, and the $\pi_{*} L_{K(1)} S$-submodule of $\pi_{*} L_{K(1)} M$ generated by $i_{*} \pi_{*} M$ is still

$$
\mathbb{F}_{p}[\sigma] \otimes E(\rho) \cup \mathbb{F}_{p}\left[\sigma, \sigma^{-1}\right] \otimes \rho,
$$

as in Remark 5.4. So, $M$ is not $L_{K(1)}$-dense.

\section{Duality}

In this section, we work in the environment described in $\S 2$. Refer to a spectrum $X$ as $L$-co-ghost-preserving if the localization of any ghost into $X$ is also a ghost. Refer to $L$ as co-ghost-preserving if every spectrum is $L$-co-ghost-preserving; $L$ is ghost-preserving if and only if it is co-ghost-preserving, so this is nothing new. Call $L$ weakly co-ghost-preserving if every finite spectrum is $L$-co-ghost-preserving. For 
our universal example, we will use the obvious dualization of the construction in $\S 2$. Let $\xi_{X}: X \longrightarrow I_{X}$ be a map to an injective $R$-module such that $\pi_{*} \xi_{X}$ is injective. Form a cofiber sequence

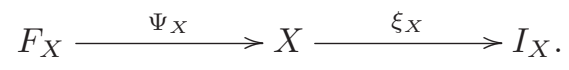

The map $\Psi_{X}$ is a universal ghost into $X$.

Proposition 7.1. Let $L$ be a localization functor, and fix an $R$-module spectrum $X$. The following statements are equivalent:

A. The spectrum $X$ is L-co-ghost-preserving.

B. The map $L \Psi_{X}$ is a ghost.

Either of the above equivalent statements is implied by

$C$. For all maps $g: Y \longrightarrow X$, the map ig is a ghost if and only if $L g$ is a ghost. If $X$ is L-local, then all of the above statements are equivalent.

Proof. It is clear that $(\mathrm{A}) \Longrightarrow(\mathrm{B})$. For the converse, simply observe that any ghost $g$ into $X$ factors through $\Psi_{X}$ since there are no non-trivial ghost maps to an injective spectrum. Hence, if $L \Psi_{X}$ is a ghost, then so is $L g$. It is also clear that (C) $\Longrightarrow(\mathrm{A})$, since $i g$ is a ghost whenever $g$ is a ghost. When $X$ is $L$-local, (A) $\Longrightarrow(\mathrm{C})$ since $i g=g$.

Remark 7.2. We have chosen the definition of co-ghost-preserving to be the more natural one, though it is not equivalent to $(\mathrm{C})$ in the above proposition, so far as we know. Another option would be to take (C) as the definition. This is equivalent to the map $L \Psi_{X}^{\prime}$ being a ghost, where $\Psi_{X}^{\prime}$ is a universal ghost into $L X$, and there is a factorization $L \Psi_{X}^{\prime} L q=L \Psi_{X}$. The issue is that when $i g$ is a ghost, it is not necessarily a ghost into $X$.

Freyd's generating hypothesis says that if $f: X \longrightarrow Y$ is a ghost map between finite spectra, then $f=0$. We include an interesting consequence of the generating hypothesis below. A map $f: Y \longrightarrow X$ is phantom if, for all $k: Z \longrightarrow Y$ with $Z$ finite, $f k=0$. A map $f$ is phantom if and only if $\pi_{*}(Z \wedge f)=0$ for all finite spectra $Z$, if and only if $\pi_{*}(Z \wedge f)=0$ for all spectra $Z$.

Proposition 7.3. If the generating hypothesis is true, then every phantom-preserving localization is weakly co-ghost-preserving.

Proof. Let $L$ be a phantom-preserving localization and let $f: Y \longrightarrow X$ be a ghost map into a finite spectrum. For any map $k: Z \longrightarrow Y$ with $Z$ finite, $f k$ is a ghost and hence trivial by the generating hypothesis. This means $f$ is phantom, so $L f$ is also phantom. But every phantom map is a ghost, so $L f$ is, in fact, a ghost.

Many localizations preserve phantom maps, by the following proposition.

Proposition 7.4. Smashing localizations preserve phantom maps.

Proof. Let $f: Y \longrightarrow X$ be a phantom map, and let $L$ be a smashing localization. Then, $\pi_{*}(Z \wedge L f)=\pi_{*}((Z \wedge L S) \wedge f)=0$ for all $Z$, hence $L f$ is phantom as well. 
In particular, if the generating hypothesis is true, then the mod $(p)$ Moore spectrum $M(p)$ is $L_{n}$-co-ghost-preserving for all $n$, though it fails to be $L_{n}$-ghost-preserving for $n \geqslant 1$.

\section{References}

[Bou79] A.K. Bousfield. The localization of spectra with respect to homology. Topology, 18(4):257-281, 1979.

[Dev90] Ethan S. Devinatz. K-theory and the generating hypothesis. Amer. J. Math., 112(5):787-804, 1990.

[Dev92] Ethan S. Devinatz. Small ring spectra. J. Pure Appl. Algebra, 81(1):11-16, 1992.

[DH95] Ethan S. Devinatz and Michael J. Hopkins. The action of the Morava stabilizer group on the Lubin-Tate moduli space of lifts. Amer. J. Math., 117(3):669-710, 1995.

[DH04] Ethan S. Devinatz and Michael J. Hopkins. Homotopy fixed point spectra for closed subgroups of the Morava stabilizer groups. Topology, 43(1):1-47, 2004.

[EKMM97] A.D. Elmendorf, I. Kriz, M.A. Mandell, and J.P. May. Rings, modules, and algebras in stable homotopy theory, volume 47 of Math. Surveys Monogr. American Mathematical Society, Providence, RI, 1997. With an appendix by M. Cole.

[Fre66] Peter Freyd. Stable homotopy. In Proc. Conf. Categorical Algebra (La Jolla, Calif., 1965), pages 121-172. Springer, New York, 1966.

[HL09] Mark Hovey and Keir Lockridge. Semisimple ring spectra. New York J. Math., 15:219-243, 2009.

[HL11] Mark Hovey and Keir Lockridge. The ghost and weak dimensions of rings and ring spectra. Israel J. Math., 182:31-46, 2011.

[HL13] Mark Hovey and Keir Lockridge. Homological dimensions of ring spectra. Homology Homotopy Appl., 15(2):53-71, 2013.

[Hov95] Mark Hovey. Bousfield localization functors and Hopkins' chromatic splitting conjecture. In The Čech Centennial (Boston, MA, 1993), volume 181 of Contemp. Math., pages 225-250. American Mathematical Society, Providence, RI, 1995.

[HP99] Mark Hovey and John H. Palmieri. The structure of the Bousfield lattice. In Homotopy Invariant Algebraic Structures (Baltimore, MD, 1998), volume 239 of Contemp. Math., pages 175-196. American Mathematical Society, Providence, RI, 1999.

[HPS97] Mark Hovey, John H. Palmieri, and Neil P. Strickland. Axiomatic stable homotopy theory. Mem. Amer. Math. Soc., 128(610):x+114, 1997.

[HS98] Michael J. Hopkins and Jeffrey H. Smith. Nilpotence and stable homotopy theory. II. Ann. of Math. (2), 148(1):1-49, 1998.

[HS99] Mark Hovey and Neil P. Strickland. Morava $K$-theories and localisation. Mem. Amer. Math. Soc., 139(666):viii+100, 1999. 
[Mil81] Haynes R. Miller. On relations between Adams spectral sequences, with an application to the stable homotopy of a Moore space. J. Pure Appl. Algebra, 20(3):287-312, 1981.

[MP12] J.P. May and K. Ponto. More Concise Algebraic Topology. Chicago Lectures in Math. University of Chicago Press, Chicago, IL, 2012. Localization, completion, and model categories.

[Rav84] Douglas C. Ravenel. Localization with respect to certain periodic homology theories. Amer. J. Math., 106(2):351-414, 1984.

[Rav86] Douglas C. Ravenel. Complex Cobordism and Stable Homotopy Groups of Spheres, volume 121 of Pure Appl. Math. Academic Press Inc., Orlando, FL, 1986.

Mark Hovey hovey@member.ams.org

Department of Mathematics, Wesleyan University, Middletown, CT 06459, USA

Keir Lockridge klockrid@gettysburg.edu

Department of Mathematics, Gettysburg College, Gettysburg, PA 17325, USA 